\title{
Genetic Analysis of Children with Dravet Syndrome in a Resource- limited Setting
}

\author{
Sorawit Viravan, M.D. ${ }^{1}$, Chutima Meesamarnpong, B.Sc. ${ }^{2}$, Wanna Thongnoppakhun, Ph.D. ${ }^{2}$, \\ Mongkol Chanvanichtrakool, M.D. ${ }^{1}$
}

'Division of Neurology, Department of Pediatrics, Faculty of Medicine Siriraj Hospital, Mahidol University, Bangkok 10700, Thailand. ${ }^{2}$ Division of Medical Genetics Research and Laboratory, Research Department, Faculty of Medicine Siriraj Hospital, Mahidol University, Bangkok 10700, Thailand.

Received 20 May 2021 • Revised 18 June 2021 • Accepted 19 June 2021 • Published online 8 September 2021

\begin{abstract}
:
Objective: To identify the common causal gene mutations in Thai children with the Dravet (DS) phenotype, using single gene analysis.

Material and Methods: The study was carried out on 20 DS patients at Siriraj Hospital, Bangkok, Thailand. Sanger sequencing of the Voltage-Gated Sodium Channel Alpha Subunit 1 (SCN1A) gene was conducted in all patients. In SCN1A-negative patients, multiplex ligation-dependent probe amplification of the SCN1A gene was performed in all cases; however, direct sequencing of the Protocadherin-19 (PCDH19) gene was analyzed in girls only.

Results: Fourteen (70.0\%) DS patients were found to carry pathogenic SCN1A mutations, with 6 novel mutations. In SCN1A-negative patients; 1 of the 4 girl patients (25.0\%) had a novel PCDH19 mutation, while none of the 6 patients had a large deletion or duplication in the SCN1A gene.

Conclusion: The SCN1A gene is the most common causative mutation in Thai children with DS phenotype. This study emphasizes the benefit of Sanger sequencing of the SCN1A gene in resource-limited countries to aid in making appropriate therapeutic decisions.
\end{abstract}

Keywords: Dravet syndrome, SCN1A, PCDH19, Thai children, Sanger sequencing

Contact: Mongkol Chanvanichtrakool, M.D.

Division of Neurology, Department of Pediatrics, Faculty of Medicine Siriraj Hospital, Mahidol University, Bangkok 10700, Thailand.

E-mail: Mongkol.cha@mahidol.edu

2021 JHSMR. Hosting by Prince of Songkla University. All rights reserved.

This is an open access article under the CC BY-NC-ND license

(http://www.jhsmr.org/index.php/jhsmr/about/editorialPolicies\#openAccessPolicy).

J Health Sci Med Res 2022;40(3):301-308 doi: 10.31584/jhsmr.2021838 www.jhsmr.org 


\section{Introduction}

Severe myoclonic epilepsy in infancy (SMEI), or Dravet syndrome (DS), is a rare developmental and epileptic encephalopathy (DEE) occurring before 1 year of age. Patients usually present with recurrent febrile seizures, unilateral clonic seizures, and prolonged febrile status epilepticus. After the first year of life, they also present with nonfebrile seizures, and multiple types of other seizures, including myoclonic seizures, absences seizures, atonic seizures, and focal seizures. ${ }^{1-3}$ Such seizures often resist treatment, and are aggravated by fever, hot weather ${ }^{4}$, vaccines $^{5}$, and sodium channel blocker agents. ${ }^{6}$

In several studies ${ }^{3,7,8}$, Voltage-Gated Sodium Channel Alpha Subunit 1 (SCN1A) mutations, as analyzed by the Sanger method, have been mainly reported in $68.4-76.7 \%$ of DS cases. In two studies ${ }^{7,9}, 12.5-13.3 \%$ of the negative cases were detected to have deletion and duplication, according to multiplex ligation-dependent probe amplification (MLPA) analysis. In addition, Protocadherin-19 (PCDH19) mutations were found in $24.4 \%$ of girls with SCN1A-negative DS. ${ }^{10}$ PCDH19 mutations are now considered to cause epilepsy in females with mental retardation (EFMR); although a few DS cases have been reported to be caused by other gene mutations; such as, to the GABRG211 $S C N 1 B^{12}$, and $S C N 2 A^{13}$ genes.

The SCN1A gene consists of 26 exons, and is located on chromosome 2q24. The type-I sodium channel is expressed in the brain and plays an important role in the firing action potential. In a rodent study, heterozygous SCN1A mutation was found to cause a reduced firing action potential in inhibitory interneurons, but not in excitatory pyramidal neurons; resulting in hyperexcitatory and seizures. ${ }^{14}$

In Thailand, a resource-limited setting, there is no previous study concerning the clinical characteristic of DS, or its causative gene among Thai Children. Hence, we aimed to identify the common causal mutations in Thai children with the DS phenotype, at Siriraj Hospital, by using the Sanger sequencing method. With the appropriate chosen patients, we also wanted to demonstrate the benefit of single gene analysis in resource-limited settings.

\section{Material and Methods}

This study represents a cross-sectional study. We reviewed the medical records of patients, aged 4 months-15 years old, with epilepsy and febrile seizure, who were followed-up at the Department of Pediatrics, Siriraj Hospital; between January 1, 2007 and March 31, 2017. Our study included all patients with the DS phenotype, identified by reviewing their previous medical records. We defined DS phenotype as infantile-onset recurrent complex febrile seizure; such as, a unilateral clonic seizure, prolonged seizure or repetitive seizures; plus at least one of the following features: multiple types of seizures (>2 seizure types), developmental delay after onset of seizure, and drug resistance. The exclusion criteria were patients with known causes of epilepsy; such as, previous cerebral insult (asphyxia, intracerebral hemorrhage, central nervous system infection), inborn error of the metabolism, brain malformation, or neurocutaneous syndrome. We reviewed the patients' demographic data, characteristics of the seizures, neuroimaging results, and electroencephalography (EEG) results from the patients' medical records. Genetic analysis was then performed in all patients. The protocol for this study was approved by the Siriraj Institutional Review Board, Faculty of Medicine Siriraj Hospital, Mahidol University, Bangkok, Thailand. Written informed consents were obtained from the gardians.

The genetic analysis was performed in patients with DS phenotype; as shown in Figure 1. DNA was extracted from the peripheral blood lymphocytes by a salting-out procedure, then amplified by polymerase chain reaction (PCR), focusing on 28 fragments covering 26 exons of the SCN1A gene (NM_001165963.4), using the published 
primers. $^{8}$ The SCN1A PCR products were then directly sequenced in both directions via Sanger sequencing. Once available, the patients' parental origins were also determined, by direct sequencing of the corresponding exons, to investigate the specified variants found in patients. In female children without SCN1A mutations, direct Sanger sequencing of the PCDH19 gene (NM_001184880.2) in both DNA strands of 11 PCR fragments covering 6 exons was also performed. Detailed information regarding the newly designed PCR primers for the $\mathrm{PCDH} 19$ gene are provided in the Supplemental Table S1. Furthermore, in cases that were negative for SCN1A point mutations, MLPA analysis of the SCN1A gene was performed to identify any large deletions or duplications. The commercially available MLPA kit (SALSA $^{\circledR}$ MLPA $^{\circledR}$ Probemix P137-C1 SCN1A;
MRC-Holland, Amsterdam, the Netherlands) was utilized, according to the manufacturer's instructions.

The identified variants were evaluated for their pathogenicity, and were classified based on a variety of supporting evidence; such as the literature, public databases, population allele frequencies, and in silico prediction tools. For missense variants, an integrated tool, VarCards, was applied for their pathogenicity evaluation based on 23 different algorithms. ${ }^{15}$ To assess the effects of the identifiable variants on the mRNA splicing process, varSEAK online (URL: https://varseak.bio/) was used. In addition, a versatile variant search engine, VarSome, was also applied. ${ }^{16}$ The variant interpretation was performed in accordance with the standards and guidelines of the American College of Medical Genetics and Genomics and the Association for Molecular Pathology. ${ }^{17}$

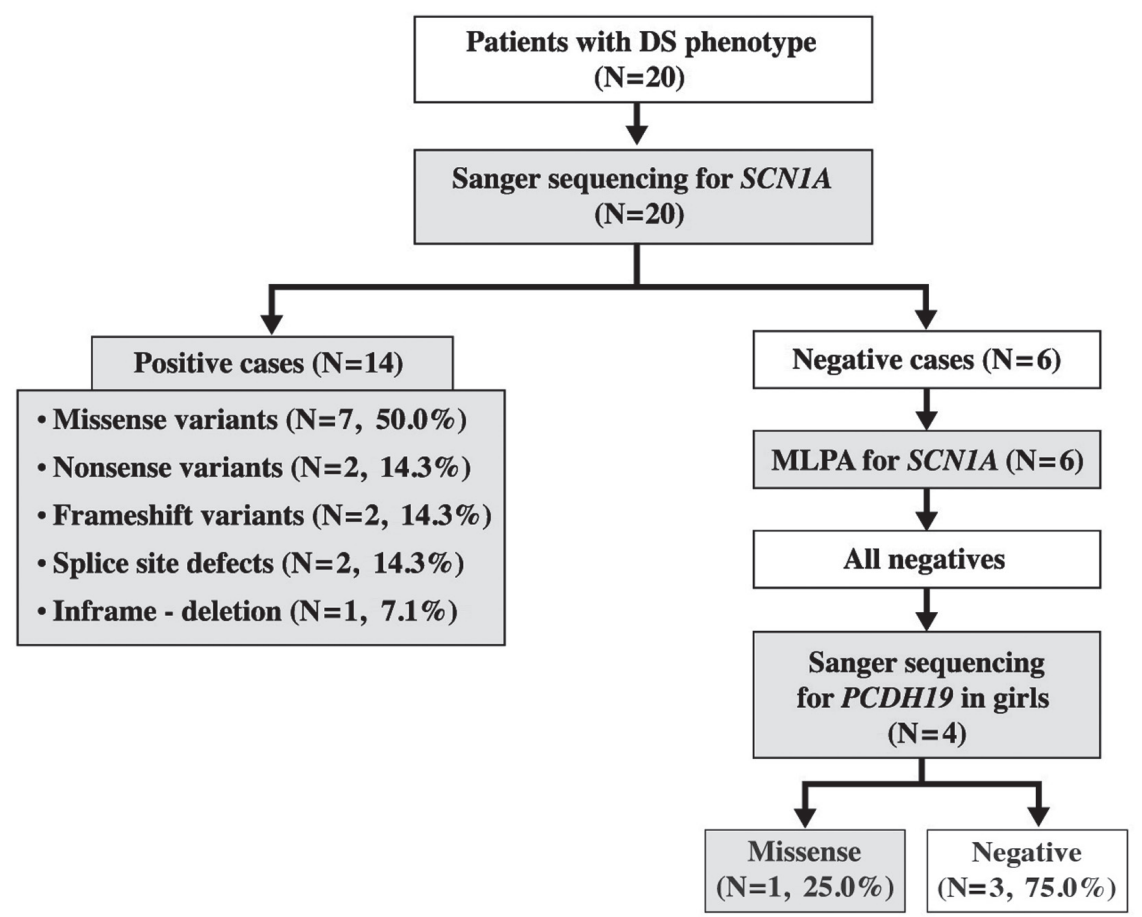

DS=Dravet syndrome, MLPA=multiplex ligation-dependent probe amplification, PCDH19=Protocadherin-19, SCN1A=Voltage-Gated Sodium Channel Alpha Subunit

Figure 1 Summarized flowchart of the genetic analysis of the patients with the Dravet syndrome phenotype 
We used descriptive statistics to analyze the demographic data and genetic testing results, and reported these by number, percentage, and mean and standard deviation for normal distributions, and median and range for non-normal distributions.

\section{Results}

We identified 369 patients with epilepsy and febrile seizures, treated at Siriraj Hospital; between 2007 and 2017: 349 patients were then excluded as they did not meet the criteria. The 20 remaining DS-phenotype patients were enrolled into this study. These patients had been previously diagnosed as drug resistant epilepsy (DRE) (65.0\%), DS (15.0\%), epilepsy (15.0\%), and Lennox-Gastaut syndrome (5.0\%).

The enrolled patients' demographic data are demonstrated in Table 1. Among the 20 enrolled patients, $14(70.0 \%)$ were males. Their median age was 5 years old (ranging from 1-14 years old). Only two patients had a history of first-degree relatives with febrile seizures. The most common clinical features were complex febrile seizure, recurrent febrile seizures, and seizures provoked by lowgrade fever, which were seen in all patients.

\section{Seizure characteristics}

The mean age of seizure onset was $5.6 \pm 1.9$ months old. The majority of seizure types were generalized tonicclonic/generalized tonic seizure or focal seizures. Eleven patients $(55.0 \%)$ had status epilepticus. Of the 14 patients who received $\mathrm{Na}$-channel blocker antiseizure medication (ASM), 13 (92.8\%) had an increased seizure frequency. The seizures were not controlled by at least two ASM in 17 patients (85\%). Other characteristics of the seizures and the aggravating factors are shown in Table 1.
Table 1 Clinical phenotype and seizure characteristics of 20 patients with Dravet syndrome

\begin{tabular}{ll}
\hline Clinical phenotype & $\mathbf{n}(\%)$ \\
\hline Age (years) (median, range) & $5,1-14$ \\
Gender (male) & $14(70.0 \%)$ \\
Family history of febrile seizure & $2(10.0 \%)$ \\
Developmental regression after seizure onset & $19(95.0 \%)$ \\
Crouch gait & $9(45.0 \%)$ \\
\hline Seizure characteristics ( $\mathbf{n}=20)$ & $\mathbf{n}(\%)$ \\
\hline Onset of seizure (months) (mean \pm SD) & $5.6 \pm 1.9$ \\
Number of seizures in first year (episodes) & $8,1-35$ \\
(median, range) & \\
Complex febrile seizure & $20(100.0 \%)$ \\
Duration >15 minutes & $8(40.0 \%)$ \\
Focal seizure (hemiclonic seizure) & $12(60.0 \%)$ \\
Recurrent seizures in 24 hours & $18(90.0 \%)$ \\
Seizure type & \\
GC/GT/GTC seizure & $18(90.0 \%)$ \\
Focal seizure & $15(75.0 \%)$ \\
Myoclonic seizure & $9(45.0 \%)$ \\
Atypical absence seizure & $6(30.0 \%)$ \\
Atonic seizure & $2(10.0 \%)$ \\
Status epilepticus & $11(55.0 \%)$ \\
Age onset of status epilepticus (months) & $13,6-101$ \\
(median, range) & $1,1-10$ \\
Number of status epilepticus (episodes) & \\
(median, range) & $13(65.0 \%)$ \\
Aggravating factor & $13(92.8 \%)$ \\
Vaccine & $6(30.0 \%)$ \\
Na-channel blocker (n=14) & $5(25.0 \%)$ \\
Hot weather & \\
Exercise & \\
\hline & \\
& \\
\hline
\end{tabular}

$\mathrm{GC}=$ generalized clonic, $\mathrm{GT}=$ generalized tonic, $\mathrm{GTC}=$ generalized tonic-clonic

\section{Investigations}

The first EEG was abnormal in 10 patients (50.0\%). Nine patients had epileptiform discharge: 7 frontal, 1 central, and 1 in the parietal region. In addition, intermittent slow activities were observed in 2 patients. None of the patients 
had photoparoxysmal response (PPR). Magnetic resonance imaging (MRI) of the brain was performed in 15 patients. Seven $(46.6 \%)$ of these revealed abnormalities, including a non-specific increased signal intensity on T2W (33.3\%), cerebellar atrophy (6.7\%), and delayed myelination (6.7\%).

\section{Genetic analysis}

Summaries of the results of the genetic analysis in this study are shown in Table 2. Pathogenic and likely pathogenic SCN1A variants were detected by the Sanger method in 14 out of $20(70.0 \%)$ patients; 6 of these were novel, as described in Table 2. All the identified SCN1A variants were in a heterozygous state, comprising 7 missense, 2 frameshift, 2 nonsense mutations, 2 splicing defects, and 1 inframe deletion.

Among the 4 girls without SCN1A mutation, one (25.0\%) was found to carry a novel heterozygous, likely pathogenic, variant in the $\mathrm{PCDH} 19$ gene. This missense variant was not found in her parents: indicating a de novo change. In the 6 patients without SCN1A mutations detected by Sanger sequencing, no large deletions or duplications in the SCN1A gene were found from MLPA analysis.

Table 2 presents the scoring of the variant classification following the 2015 American College of Medical Genetics and Genomics (ACMG) and the Association for Molecular Pathology (AMP) standards and guidelines, shown as abbreviations; such as, pathogenic-very strong (PVS), pathogenic-strong (PS), pathogenic-moderate (PM), and pathogenic-supporting (PP). Variant interpretations were then performed by integration of all the met criteria. For example, SCN1A:c.429_430delGT (p.Phe144Tyrfs*5) was interpreted as pathogenic, because of PVS1 (null variant, frameshift deletion), PM2 (absent from controls), PM6 (assumed de novo), and PP4 (phenotype specific for DS). Alternatively, PCDH19:c.1124A>T (p.Asp 375Val) was interpreted as likely pathogenic, due to PS2 (approved de novo), PM2 (absent from the controls), PP3 (pathogenic computational predictions), and PP4 (phenotype specific for DS).

\section{Discussion}

The causative gene and mutation types of DS in our ethnic group are similar to those in other studies; which reported that $68.4-76.7 \%$ of patients had positive SCN1A mutations. ${ }^{3,7}$ Missense mutations were the most common, followed by nonsense mutations, frameshift mutations, splicing defects, and inframe mutations, respectively. Large deletions or duplications in SCN1A, analyzed by the MLPA method, were not found in our SCN1A mutation-negative patients. This may be due to our lower number of DS patients, which contrasts with previous studies that reported an incidence of $12.5-13.3 \% .^{7,9}$ The PCDH19 mutation was also detected in 1 female patient with negative SCN1A mutation $(25.0 \%)$, which is the same as in a previous study. ${ }^{10}$

Before the availability of SCN1A gene test for DS, most of our patients were misdiagnosed with DRE; which misled to ASM treatment, such as $\mathrm{Na-channel} \mathrm{blockers,}$ causing seizure worsening. After the diagnosis was confirmed with the SCN1A gene test, it could help physician avoid aggravated ASM and choose an appropriate ASM; such as, sodium valproate, clobazam, stiripentol leading to improvement of seizure control. This highlights the importance of performing genetic tests on DS patients, and also encourages physicians to perform genetic tests. In the future, the treatment of epilepsy should be changed so as to be based on genetic results. Nowadays, advanced genetic tests; such as, whole exome sequencing (WES) and epilepsy multigene panel tests, are available worldwide, and have demonstrated a diagnostic benefit for DEE. They are also more useful now in identifing various causative genes; especially in atypical presentations of DS. ${ }^{18}$

However, in Thailand these advanced genetic tests are expensive as well as less available. They also require experienced scientists, and have a longer turnaround 


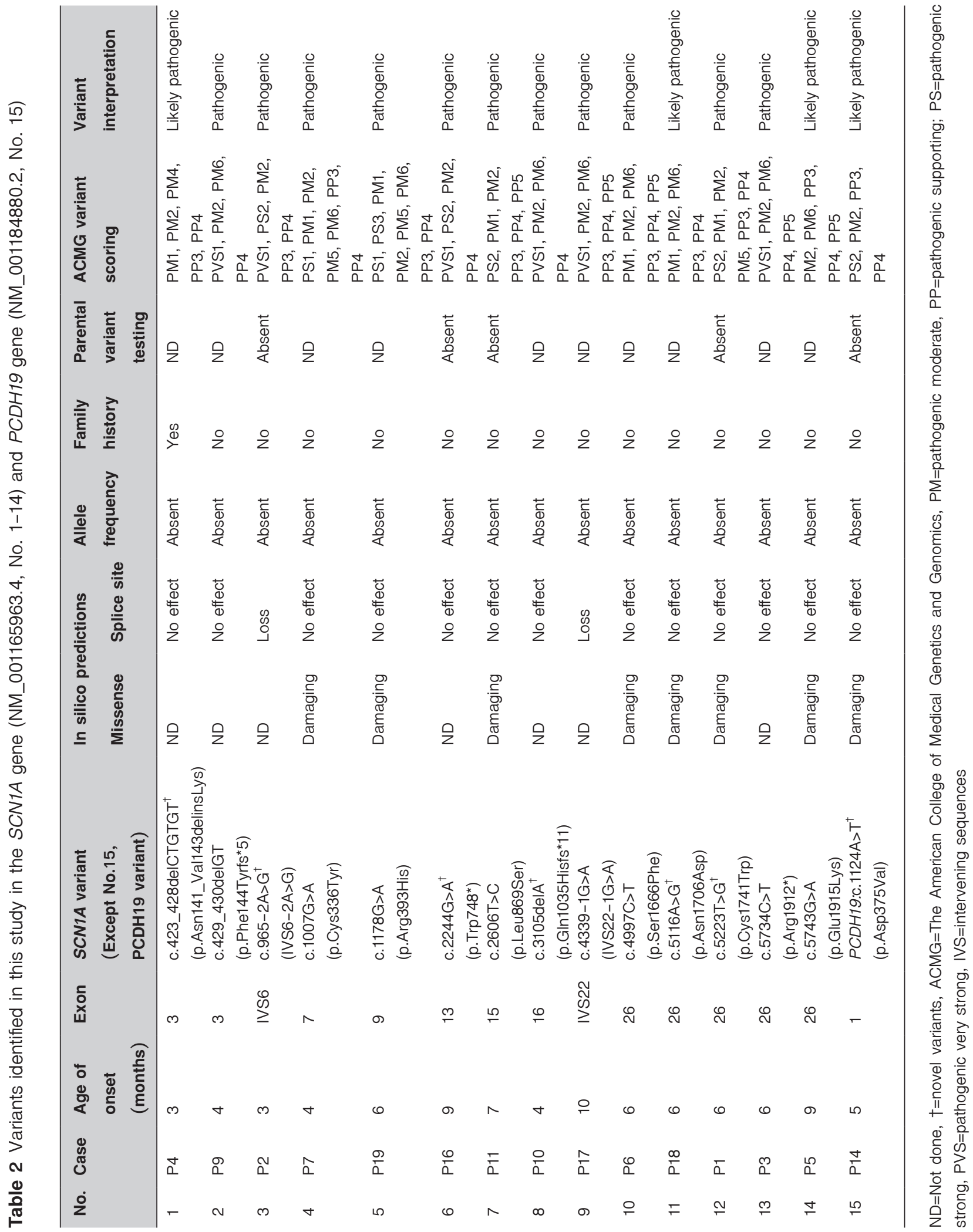


time (up to 6 months). On the other hand, DS has more distinctive clinical phenotypes, and our data has shown that the majority of DS is caused by SCN1A gene mutation in the Thai population. Moreover, the SCN1A single gene test is more economical, and has a shorter turnaround time (up to 3 months), with the same detection rate as in other methods. Therefore, in resource-limited countries, single analysis of the $S C N 1 A$ gene is a reasonable choice as the first genetic test in patients with the DS phenotype. After this, WES or epilepsy multigene panel testing may be the next step of investigation, if the results returned negative.

The clinical characteristics of seizures in our ethnic group were similar to that of previous studies. ${ }^{2,3,19,20}$ The most common symptoms of DS are cluster, prolonged, fever-related, intractable seizures, and developmental delay after the onset of seizure.

In the aspect of our investigations, half of the patients had a normal EEG at the first study. The abnormalities found on the EEGs; including, focal spike, a multifocal spike predominating over the frontal region, and a slow background, are similar to those found in the study by Lee. ${ }^{21}$ However, our patients had no PPR on their EEG compared with $30.7-34.7 \%$ in other studies. ${ }^{19,22}$ The incidence of cerebral and cerebellar atrophy detected in our study is lower than in previous studies. ${ }^{22,23}$ The abnormalities in brain MRI mainly depend on the age of the patient, and in our study, the mean age for MRI examinations was lower than in Striano's study ${ }^{23}$; therefore, obvious brain atrophy may not have been found in our patients.

Single gene analysis has limitations, as our study only identified the main causative genes of DS; such as, SCN1A and PCDH19 genes. Therefore, we could not identify other causative genes of DS; such as, GABRG2 $2^{11}, S C N 1 B^{12}$, and $S C N 2 A^{13}$ genes. Therefore, WES or an epilepsy multipanel would be the next step of testing.

\section{Conclusion}

The single gene analysis of SCN1A could identify the majority of causative genes for the DS phenotype in Thai children. Therefore, in resource-limited countries, it would be reasonable to be used as the first genetic test in patients with the typical DS phenotype. This also emphasizes the benefit of Sanger sequencing of the SCN1A gene, which can help lead to appropriate therapeutic decisions. Additionally, as EFMR can mimic the DS phenotypes, $P C D H 19$ mutation testing should be considered in female patients with a mimic of the DS phenotype.

\section{Acknowledgement}

The authors are grateful to all the patients, and their gardians who participated in this study.

\section{Funding sources}

This work was supported by Siriraj Research and Development Fund [R016032019] as well as by the Siriraj Core Research Facility, Faculty of Medicine Siriraj Hospital, Mahidol University.

\section{Conflict of interest}

None of the authors have any conflict of interest to declare.

\section{References}

1. Akiyama M, Kobayashi K, Ohtsuka Y. Dravet syndrome: a genetic epileptic disorder. Acta Med Okayama 2012;66:369-76.

2. Dravet C. The core Dravet syndrome phenotype. Epilepsia 2011;52(Suppl 2):3-9.

3. Xu X, Zhang $Y$, Sun $H$, Liu X, Yang X, Xiong $H$, et al. Early clinical features and diagnosis of Dravet syndrome in 138 Chinese patients with SCN1A mutations. Brain Dev 2014;36: 676-81. 
4. Moreno de Flagge N. Simple febrile seizure, complex seizure, generalized epilepsy with febrile seizure plus, FIRES and new syndromes. Medicina (B Aires) 2013;73(Suppl 1):S63-70.

5. Berkovic SF, Harkin L, McMahon JM, Pelekanos JT, Zuberi $\mathrm{SM}$, Wirrell EC, et al. De-novo mutations of the sodium channel gene SCN1A in alleged vaccine encephalopathy: a retrospective study. Lancet Neurol 2006;5:488-92.

6. Guerrini R, Dravet C, Genton P, Belmonte A, Kaminska A, Dulac O. Lamotrigine and seizure aggravation in severe myoclonic epilepsy. Epilepsia 1998;39:508-12.

7. Depienne C, Trouillard O, Saint-Martin C, Gourfinkel-An I, Bouteiller D, Carpentier W, et al. Spectrum of SCN1A gene mutations associated with Dravet syndrome: analysis of 333 patients. J Med Genet 2009;46:183-91.

8. Claes L, Del-Favero J, Ceulemans B, Lagae L, Van Broeckhoven $C$, De Jonghe P. De novo mutations in the sodium-channel gene SCN1A cause severe myoclonic epilepsy of infancy. Am J Hum Genet 2001;68:1327-32.

9. Marini C, Scheffer IE, Nabbout R, Mei D, Cox K, Dibbens LM, et al. SCN1A duplications and deletions detected in Dravet syndrome: implications for molecular diagnosis. Epilepsia 2009;50:1670-8.

10. Depienne C, Bouteiller D, Keren B, Cheuret E, Poirier K, Trouillard $\mathrm{O}$, et al. Sporadic infantile epileptic encephalopathy caused by mutations in $\mathrm{PCDH} 19$ resembles Dravet syndrome but mainly affects females. PLoS Genet 2009;5:e1000381.

11. Shi X, Huang MC, Ishii A, Yoshida S, Okada M, Morita K, et al. Mutational analysis of GABRG2 in a Japanese cohort with childhood epilepsies. J Hum Genet 2010;55:375-8.

12. Patino GA, Claes LR, Lopez-Santiago LF, Slat EA, Dondeti $\mathrm{RS}$, Chen $\mathrm{C}$, et al. A functional null mutation of SCN1B in a patient with Dravet syndrome. J Neurosci 2009;29:10764-78.

13. Shi X, Yasumoto S, Kurahashi H, Nakagawa E, Fukasawa T, Uchiya S, et al. Clinical spectrum of SCN2A mutations. Brain Dev 2012;34:541-5.
14. Yu FH, Mantegazza M, Westenbroek RE, Robbins CA, Kalume $\mathrm{F}$, Burton KA, et al. Reduced sodium current in GABAergic interneurons in a mouse model of severe myoclonic epilepsy in infancy. Nat Neurosci 2006;9:1142-9.

15. Li J, Shi L, Zhang K, Zhang Y, Hu S, Zhao T, et al. VarCards: an integrated genetic and clinical database for coding variants in the human genome. Nucleic Acids Res 2018;46:D1039-D48.

16. Kopanos C, Tsiolkas V, Kouris A, Chapple CE, Albarca Aguilera $\mathrm{M}$, Meyer R, et al. VarSome: the human genomic variant search engine. Bioinformatics 2019;35:1978-80.

17. Richards S, Aziz N, Bale S, Bick D, Das S, Gastier-Foster $\mathrm{J}$, et al. Standards and guidelines for the interpretation of sequence variants: a joint consensus recommendation of the American College of Medical Genetics and Genomics and the Association for Molecular Pathology. Genet Med 2015; 17:405-24.

18. Minardi R, Licchetta L, Baroni MC, Pippucci T, Stipa C, Mostacci $B$, et al. Whole-exome sequencing in adult patients with developmental and epileptic encephalopathy: It is never too late. Clin Genet 2020;98:477-85.

19. Hattori J, Ouchida M, Ono J, Miyake S, Maniwa S, Mimaki N, et al. A screening test for the prediction of Dravet syndrome before one year of age. Epilepsia 2008;49:626-33.

20. Oguni H, Hayashi K, Awaya Y, Fukuyama Y, Osawa M. Severe myoclonic epilepsy in infants--a review based on the Tokyo Women's Medical University series of 84 cases. Brain Dev 2001;23:736-48.

21. Lee HF, Chi CS, Tsai CR, Chen CH, Wang CC. Electroencephalographic features of patients with SCN1A-positive Dravet syndrome. Brain Dev 2015;37:599-611.

22. Caraballo RH, Fejerman N. Dravet syndrome: a study of 53 patients. Epilepsy Res 2006;70(Suppl 1):S231-8.

23. Striano P, Mancardi MM, Biancheri R, Madia F, Gennaro E, Paravidino $\mathrm{R}$, et al. Brain MRI findings in severe myoclonic epilepsy in infancy and genotype-phenotype correlations. Epilepsia 2007;48:1092-6. 